\title{
Assessment of the Effects of Scatterer Size Distributions on Effective Scatterer Diameter Estimates
}

\author{
Roberto J. Lavarello ${ }^{*}{ }^{\dagger}$ and Michael L. Oelze* \\ * Bioacoustics Research Laboratory, Department of Electrical and Computer Engineering \\ University of Illinois at Urbana-Champaign, Urbana, IL 61801 \\ Email: lavarell@illinois.edu \\ † Laboratorio de Imágenes Médicas, Departmento de Ingeniería \\ Pontificia Universidad Católica del Perú, San Miguel, Lima 32, Perú
}

\begin{abstract}
Scattering models used in most quantitative ultrasound studies assume distributions of identical scatterers. However, actual tissues may exhibit multiple levels of spatial scales. Therefore, the objective of the present study was to use both simulations and experiments to analyze the effects of scatterer size distributions when using a fluid-sphere model for estimating effective scatterer diameter (ESD). Simulations were conducted with populations of scatterers with uniformly distributed sizes within $[25,100],[25,50],[50,100]$, and $[50,75]$ $\mu \mathrm{m}$. Simulated backscatter coefficients (BSCs) used as inputs for the ESD estimator were obtained using two methods: (1) using portions of the theoretical BSC with different center frequencies between 1 and $40 \mathrm{MHz}$ and $100 \%$ fractional bandwidth, and (2) processing simulated radiofrequency data from computer phantoms using $\mathrm{f} / 4$ transducers with center frequencies of 3,6 , 12, 24, and $36 \mathrm{MHz}$ and $100 \%$ fractional bandwidth. Experiments were conducted using a gelatin phantom with Sephadex spheres ranging in diameter from $30 \mu \mathrm{m}$ to $140 \mu \mathrm{m}$ and 3.5, 7.5, 10, and $13 \mathrm{MHz}$ focused transducers. ESD estimates obtained with both simulation methods were approximately inversely proportional to frequency and mostly independent of the underlying scatterer size distribution for sufficiently high analysis frequencies. For frequencies higher than $13 \mathrm{MHz}$ the ESD estimates were below $50 \mu \mathrm{m}$ for all considered size distributions even though two of them had no scatterers smaller than $50 \mu \mathrm{m}$. Further, the asymptotic behavior of ESD vs. frequency estimates was also observed experimentally. The results of this work highlight some of the effects of continuous scatterer size distributions when obtaining ESD estimates, and challenge the hypothesis that different frequency ranges are more sensitive to different spatial scales when using a single-size scattering model. Although the results presented here are not necessarily universal and most likely will be affected by the actual size distribution and frequency-dependent BSC of the individual scatterers, this work suggests that caution must be exerted when interpreting ESD estimates at different frequency ranges. This work was supported by a grant from the NIH R21CA139095.
\end{abstract}

\section{Motivation}

Quantitative ultrasound imaging based on backscattering coefficients (referred to in this work as QUS) has proven potential for tissue characterization. Using QUS, microstructural information from samples is obtained by fitting estimated backscattering coefficients (BSCs) to theoretical models of scattering. A commonly used scattering model in the study of both physical [1] and biological [2] phantoms is to assume BSCs are produced by discrete scatterers embedded in an otherwise homogeneous background. Under this assumption, the quantification of microstructural properties is performed by fitting estimated BSCs to a model corresponding to an ensemble of discrete scatterers of certain effective scatterer diameter (ESD). However, the convergence of ESD estimates in the presence of scatterer populations of different sizes has received only limited attention. Roberjot et al. [3] and Insana and Hall [4] studied through experiments the effects of discrete (i.e., Bernoulli) and continuous (i.e., Gaussian) scatterer size distributions, respectively, on ESD estimates. Roberjot et al. concluded that different frequency ranges were more sensitive to different spatial scales, and that in particular higher frequencies were more sensitive to smaller scatterers. Insana and Hall studied the effects of the ratio of the standard deviation $\sigma$ and mean $\mu$ values of the size distribution when obtaining ESD estimates, and observed that ESD estimates changed as a function of the $k a$ range used for the estimation. Furthermore, they observed that when $k a \approx 0.8$ the ESD estimate corresponded to $\mu$ with a bias of less than $10 \%$ independently of the $\sigma / \mu$ ratio. Although frequency dependent effects of scatterer size distributions on ESD estimates have been reported, a clear description of the effects of continuous size distributions on ESD estimates using different frequency scales is still lacking. The work in [3] only considered one size distribution as a basis for all the reported conclusions. The work in [4] used Gaussian size distributions, which complicates the ESD estimate analysis because all possible scatterers sizes were present in the imaging targets. Therefore, the goal of the present work is to determine if different frequency ranges are indeed more sensitive to different spatial scales when using discrete spherical scatterers with continuous scatterer size distributions of finite support.

\section{Methodology}

\section{A. Backscattering by a multiple-sized scatterer population}

The exact analytic solution to the scattering of a plane wave of wave number $k$ by a fluid sphere of radius $a$, compressibility 
$\kappa$, and density $\rho$ embedded in a fluid medium has been previously reported in the literature [5]. Under weak scattering assumptions, the backscattering cross-section (BCS) $\sigma(k, a)$ can be written as [6]

$$
\sigma(k, a)=\frac{4 \pi a^{2}}{9}\left(\gamma_{\kappa}-\gamma_{\rho}\right)^{2}(k a)^{4}\left(\frac{3}{2 k a} j_{1}(2 k a)\right)^{2},
$$

where $\gamma_{\kappa}=\frac{\kappa-\kappa_{0}}{\kappa_{0}}$ and $\gamma_{\rho}=\frac{\rho-\rho_{0}}{\rho}$ are the fractional changes in $\kappa$ and $\rho$, respectively, and $j_{1}(\cdot)$ is the first-order spherical Bessel function. One can now consider the situation where ultrasound is scattered by an ensemble of $\beta$ scatterers per unit of volume distributed spatially at random in an otherwise homogeneous, fluid medium. Assuming no multiple scattering among the individual scatterers, and neglecting the effects of coherent scattering, the theoretical differential backscattering coefficient (BSC) $\eta(k)_{t h}$ can be expressed as

$$
\eta(k)_{t h}=\frac{\beta}{4 \pi} \int_{0}^{\infty} p(a) \sigma(k, a) d a,
$$

where $p(a)$ is the probability distribution function (PDF) of $a$.

\section{B. BSC estimates from pulse-echo data}

In order to obtain BSC estimates, an acoustic aperture receives pulse-echo pressure waveforms $s_{m}(t)$ when located at positions $y=y_{m}$. The BSC can be estimated from the backscattered data from a region of interest (ROI) gated axially between depths $(F-\Delta z / 2)$ and $(F+\Delta z / 2)$ using a rectangular window, where $F$ is the transducer's focal depth and $\Delta z$ is the gate length. The normalized backscattered power spectrum $\bar{S}(k)$ is defined here as

$$
\bar{S}(k)=\gamma^{2} \frac{\left\langle\left|S_{m}(k)\right|^{2}\right\rangle}{\left|S_{0}(k)\right|^{2}} F(k),
$$

where $\left|S_{0}(k)\right|^{2}$ is a reference power spectrum obtained from a reflection off a planar surface of known pressure reflection coefficient $\gamma,\left\langle\left|S_{m}(k)\right|^{2}\right\rangle$ is the average of the power spectra of several adjacent, gated scan lines $s_{m}(t)$, and $F(k)$ is a function that compensates for attenuation effects [7]. Following the method in [8], the estimated BSCs $\eta(k)_{e s t}$ can be calculated as

$$
\begin{aligned}
& \eta(k)_{e s t}=2.174\left|D_{\text {ref }}(k)\right| \frac{F^{2}}{A_{0} \Delta z} \bar{S}(k) \\
& D_{r e f}(k)=\exp \left(-i G_{p}\right)\left[J_{0}\left(G_{p}\right)+i J_{1}\left(G_{p}\right)\right]-1,
\end{aligned}
$$

where $A_{0}=\pi R^{2}$ and $G_{p}=k R^{2} / 2 F$ are the aperture area and pressure gain factor of the transducer of radius $R$, respectively, and $J_{m}(\cdot)$ is the $m$-th order Bessel function.

\section{Effective scatterer diameter estimation}

Microstructural information about the illuminated region can be obtained from BSC estimates $\eta(k)_{e s t}$. ESD estimates were obtained by minimizing the function [6], [9]

$$
\begin{aligned}
\mathrm{ESD} & =2 \underset{a}{\arg \min } \int_{k_{\min }}^{k_{\max }}(X(k, a)-\bar{X})^{2} d k \\
X(k, a) & =10 \log _{10}\left(\eta(k)_{e s t} / \sigma(k, a)\right),
\end{aligned}
$$

where $\bar{X}$ is the mean value of $X(f)$ within the wave number analysis bandwidth $k \in\left[k_{\min }, k_{\max }\right]$.

\section{ESD estimation simulations}

Populations of scatterers with uniformly distributed sizes were studied through simulations. Scatterer diameter ranges of $[25,100],[25,50],[50,100]$, and $[50,75] \mu \mathrm{m}$ were considered. The effects of scatterer size distributions were studied by conducting simulations using two methods:

1) Method 1: In the first method, (2) was used to calculate $\eta(k)_{t h}$ for several frequencies between 0 and $60 \mathrm{MHz}$ using the $p(a)$ that corresponds to a uniform distribution. The evaluation of (2) was performed using numerical quadrature integration. Afterwards, ESD estimates were obtained using (5) with $\eta(k)_{e s t}=\eta(k)_{t h}$. ESD estimates at different frequencies $f_{0}$ were obtained by analyzing the synthetic BSCs between $f_{\min }=0.5 f_{0}$ and $f_{\max }=1.5 f_{0}$, i.e., assuming an imaging system with $100 \%$ useable fractional bandwidth.

2) Method 2: The second method consisted of simulating radio frequency data and using (4) to calculate $\eta(k)_{e s t}$ for several ROIs of axial and lateral sizes of $16 \lambda$ (with $\lambda$ calculated using the transducer center frequency) and 4 lateral beamwidths, respectively. The mean and standard deviation of the estimated ESD values obtained with simulated $f / 4$ transducers of center frequencies equal to $3,6,12,24$, and 36 $\mathrm{MHz}$ and $100 \%-6 \mathrm{~dB}$ fractional bandwidth were calculated. Special care was taken to ensure the scatterers did not overlap when generating the computer phantoms used for the rf data simulations. The number of scatterers per resolution cell was set to 80 considering the $3 \mathrm{MHz}$ simulated transducer, with the same phantoms being imaged with all simulated transducers to isolate the effects of different imaging frequency ranges.

\section{E. ESD estimation experiments}

Experimental results were obtained by constructing a 250 bloom-strength, Type-B gelatin (Rousselot Inc., Dubuque, IA) phantom. The background gelatin mixture consisted of $12 \%$ w/w gelatin powder, $87 \%$ de-ionized water, and $1 \%$ Germall Plus. Scattering was produced using Sephadex spheres (Sephadex G-25 Fine, GE Healthcare) at a concentration of 2 grams of dry spheres per $400 \mathrm{ml}$ of gelatin mixture. A portion of the gelatin plus Sephadex mixture was preserved, sliced, and imaged using an optical microscope for sphere size determination. The scatterer size PDF estimate is given in Fig. 1.

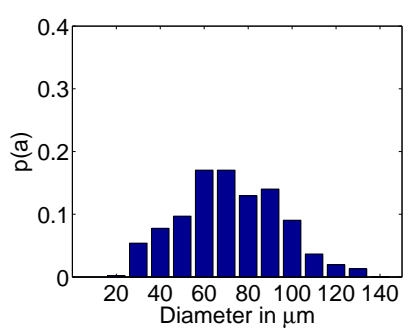

Fig. 1. Scatterer size PDF $p(a)$ corresponding to the phantom used for the experimental BSC measurements. 

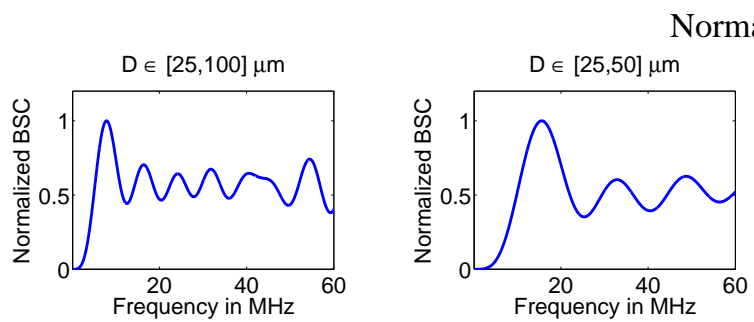

Normalized BSC
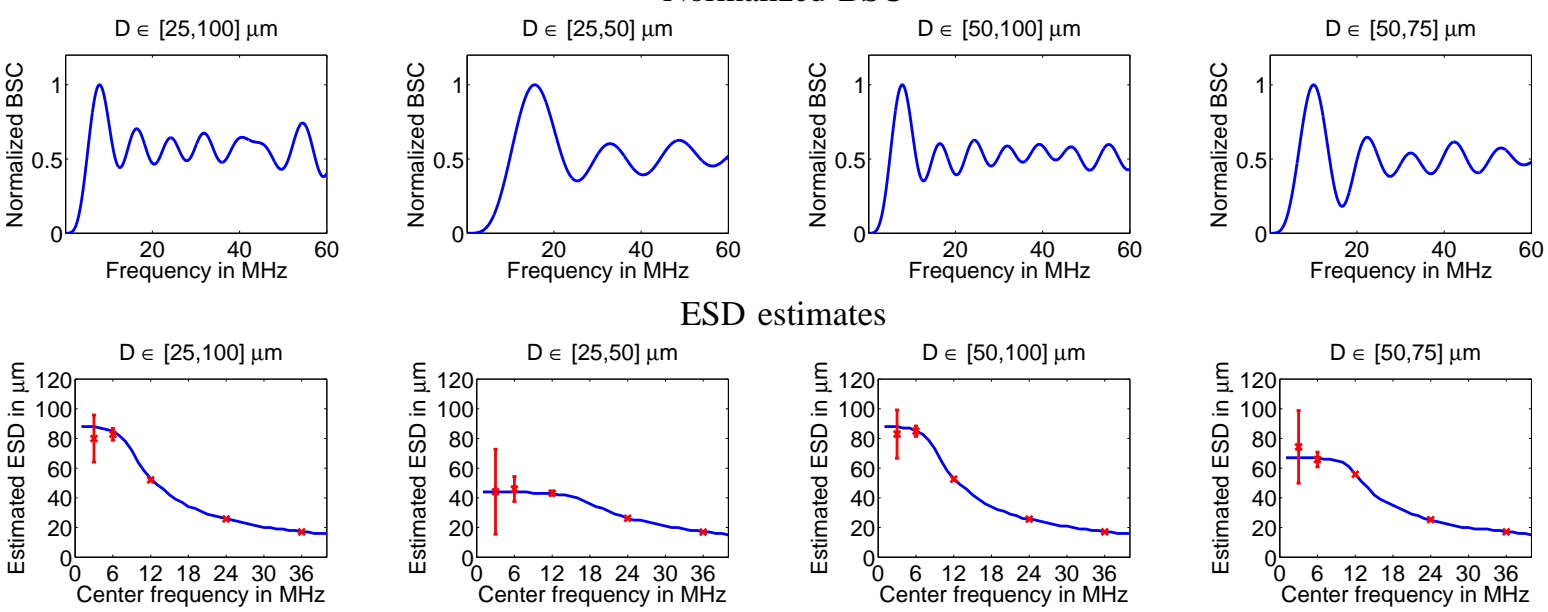

SD estimates

Fig. 2. Simulation results using a uniform scatterer size distribution. Top row: normalized BSCs as predicted by (2). Bottom row: ESD estimates obtained using methods 1 (solid line) and 2 (star marks with error bars).

The density and speed of sound of the gelatin mixture were measured to be $1.02 \mathrm{~g} / \mathrm{ml}$ and $1.54 \mathrm{~mm} / \mu \mathrm{s}$, respectively. The mass density of Sephadex was measured to be $1.3 \mathrm{~g} / \mathrm{ml}$. In this work it was assumed no sound speed contrast existed between the Sephadex spheres and the gelatin background.

Four different transducers with nominal center frequencies of $5 \mathrm{MHz}, 7.5 \mathrm{MHz}, 10 \mathrm{MHz}$, and $13 \mathrm{MHz}$ respectively, were used to scan the phantom. All transducers had fractional bandwidths between 0.9 and 1.05. A total of 441 scan lines were obtained by translating a transducer over an area of $4 \mathrm{~cm}^{2}$. Spectra from segments of length $\Delta z=15 \lambda$ centered around the transducer focus, with $\lambda$ calculated using the nominal transducer center frequency, were obtained for all 441 scan lines. All 441 estimated spectra were combined to obtain an average BSC curve per transducer. Attenuation compensation was performed using Ref. [7], Eq. (16) with the phantom's attenuation coefficient estimated using through-transmission measurements with an $\mathrm{f} / 4,7.5 \mathrm{MHz}$ transducer and fitted to a cubic polynomial.

\section{RESULTS}

\section{A. Simulation results}

The simulation results are presented in Fig. 2. The top row presents the BSCs corresponding to the scatterer populations (normalized to a maximum value of one for the presented frequency range) as predicted by (2) and the bottom row presents the ESD estimates using method 1 (solid line) and method 2 (star marks with error bars). For all simulations there was an agreement between the ESD estimates predicted by both simulation methods. It can be observed that both simulation methods predicted a decrease of the ESD estimates for increasing center frequency of the analysis range. Consistent with observations reported in the literature, the variance of the ESD estimates obtained using simulation method 2 increased with decreasing analysis frequencies as the dominant $k a$ values were reduced below unity [4], [10].

\section{B. Experimental results}

The experimental results are presented in Fig. 3. In the first column of Fig. 3 the normalized experimental BSC obtained using the $5 \mathrm{MHz}$ (solid line), 7.5 MHz (dashdotted line), $10 \mathrm{MHz}$ (dashed line), and $13 \mathrm{MHz}$ (dotted line) transducers, together with the theoretical BSC (thick dotted line) predicted using (2) are presented. The theoretical BSC curve was obtained using (2) together with (1) and the PDF reported in Fig. 1. The agreement between the theoretical $\eta_{t h}(k)$ and experimental $\eta_{e s t}(k)$ curves was quantified by calculating the mean and standard deviation of the log-error $10 \log _{10}\left(\eta_{\text {est }}(k) / \eta_{t h}(k)\right)$. The log-error mean and standard deviation values were $0.96 \mathrm{~dB}$ and $0.37 \mathrm{~dB}$, respectively. Therefore, both the magnitude and frequency dependence of the experimental and theoretical BSC curves were in very good agreement. The ESD estimates obtained with the $5 \mathrm{MHz}$ (star), $7.5 \mathrm{MHz}$ (pentagon), $10 \mathrm{MHz}$ (square), and $13 \mathrm{MHz}$ (diamond) transducers, together with the theoretical curve (dashed line) predicted using method 1 from Section II-D, are presented in the second column of Fig. 3. As expected from the agreement between theoretical and experimental BSC curves, the experimental and theoretical ESD estimates were in very good agreement.

\section{DISCUSSION}

The ESD curves corresponding to the four populations studied in Section III-A are presented together in Fig. 4. It can be observed that all curves closely approached a common asymptotic behavior for high enough frequency values. It is hypothesized here that the asymptotic behavior was due to the estimator locking onto the first lobe of $\sigma(k, a)$, i.e., the region around the first maximum of $\sigma(k, a)$ from (1) at $k a \approx 1.37$. This situation arises from the inability of the single-size $\sigma(k, a)$ to reproduce the expected BSC generated by populations of scatterers of different sizes for high enough frequencies. Insana and Hall [4] also observed and discussed 

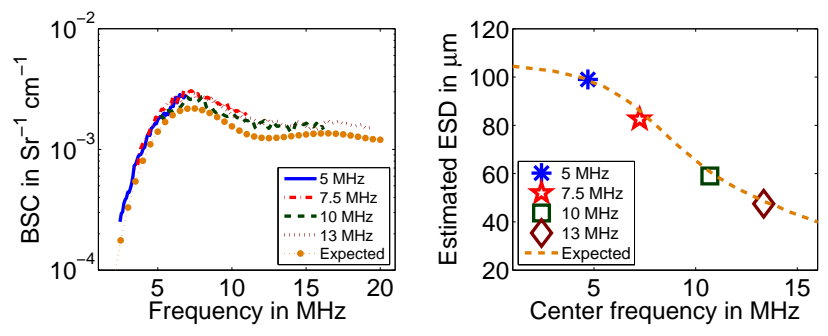

Fig. 3. Experimental results corresponding to the Sephadex sphere phantom. First column: experimental BSC curves obtained with the 5, 7.5, 10, and 13 $\mathrm{MHz}$ transducers. For comparison, the normalized BSC predicted by theory is also presented. Second column: estimated ESDs obtained with the 5, 7.5, 10 , and $13 \mathrm{MHz}$ transducers, together with the theoretical curve predicted using method 1 from Section II-D.

this estimation ambiguity when using oscillatory scattering models in the context of ESD estimation with noisy data. Insana and Hall argued in [4] the flat frequency response of $\sigma(k, a)$ around its first lobe minimized the variance of $\log \left(\eta(k)_{\text {est }} / \sigma(k, a)\right)$. The diameter $D_{A S}$ that corresponds to locking onto the first lobe of $\sigma(k, a)$ as a function of the center frequency $f$ of the analysis range is given by

$$
D_{A S} \approx \frac{2000 \times 1.37 c_{0}}{2 \pi} \frac{1}{f}=\frac{654}{f},
$$

where $c_{0}$ is the speed of sound in the background (considered here to be $1.5 \mathrm{~mm} / \mu \mathrm{s}), D_{A S}$ is given in $\mu \mathrm{m}$, and $f$ is given in MHz. The asymptotic curve corresponding to (6) is also plotted in Fig. 4 for comparison. It can be observed that for both distributions, the asymptotic curve agrees very well with the ESD estimates for sufficiently high analysis frequencies.

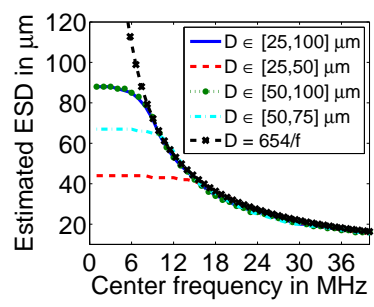

Fig. 4. ESD estimates when using uniform scatterer size distributions.

Given that the asymptotic behavior of ESD curves is almost independent of the actual scatterer size distribution, these results suggest that ESD estimates may fail to represent actual microstructural properties of the analyzed ROIs if scattering is described using a single scatterer model. In particular, Fig. 4 showed that at $36 \mathrm{MHz}$ the estimated ESDs were around 20 $\mu \mathrm{m}$ for all the analyzed distributions even though the smallest scatterers for two of the four analyzed populations were $50 \mu \mathrm{m}$ in diameter. Further, for all simulations the standard deviations of the ESD estimates using method 2 were less than $4 \%$ of the corresponding mean ESD values at $12 \mathrm{MHz}$ and above, which could erroneously be interpreted as a sign of robust estimations. More importantly, the results presented in Section III-B suggest that the trends observed in simulations may also be observed in experiments. In particular, the experimental
ESD vs. analysis frequency curve in the first column of Fig. 3 exhibited the same inverse frequency dependence observed in simulations.

\section{CONCLUSION}

The results presented in this work highlight some of the effects of continuous scatterer size distributions when obtaining ESD estimates. In general, ESD estimates were found to change as a function of the frequency range used for the size estimation. It was observed that for high frequencies the ESD estimates reached asymptotic values almost independent of the actual scatterer size distribution. Therefore, these results challenge the hypothesis that different frequency ranges are more sensitive to different spatial scales when using a single-size scattering model. Although the results presented here are not necessarily universal and most likely will be affected by the actual size distribution, frequency dependent BCS of the individual scatterers, and the actual algorithm used for ESD estimation, this work suggests that caution must be exerted when analyzing and interpreting ESD estimates over different frequency ranges. This work was supported by NIH Grant R21CA139095.

\section{REFERENCES}

[1] K. A. Wear, T. A. Stiles, G. R. Frank, E. L. Madsen, F. Cheng, E. J. Feleppa, C. S. Hall, B. S. Kim, P. Lee, W. D. O’Brien, Jr., M. L. Oelze, B. I. Raju, K. K. Shung, T. A. Wilson and J. R. Yuan, "Interlaboratory Comparison of Ultrasonic Backscatter Coefficient Measurements from 2 to 9 MHz," vol. 24, no. 9, pp. 1235-1250, September 2005.

[2] R. E. Baddour, M. D. Sherar, J. W. Hunt, G. J. Czarnota, and M. C. Kolios, "High-frequency ultrasound scattering from microspheres and single cells," Journal of the Acoustical Society of America, vol. 117, no. 2, pp. 934-943, February 2005.

[3] V. Roberjot, S. L. Bridal, P. Laugier, and G. Berger, "Absolute backscatter coefficient over a wide range of frequencies in a tissue-mimicking phantom containing two populations of scatterers," IEEE Transactions on Ultrasonics, Ferroelectrics, and Frequency Control, vol. 43, no. 5 , pp. 970-978, September 1996.

[4] M. F. Insana and T. J. Hall, "Parametric ultrasound imaging from backscatter coefficient measurements: Image formation and interpretation," Ultrasonic Imaging, vol. 12, no. 4, pp. 245-267, October 1990.

[5] V. C. Anderson, "Sound scattering from a fluid sphere," Journal of the Acoustical Society of America, vol. 22, no. 4, pp. 426-431, July 1950.

[6] M.F. Insana, R.F. Wagner, D.G. Brown, and T.J. Hall, "Describing smallscale structure in random media using pulse-echo ultrasound," Journal of the Acoustical Society of America, vol. 87, no. 1, pp. 179-192, January 1990.

[7] M. L. Oelze and W. D. O’Brien, Jr., "Frequency-dependent attenuationcompensation functions for ultrasonic signals backscattered from random media,' Journal of the Acoustical Society of America, vol. 111, no. 5, pp. 2308-2319, May 2002.

[8] X. Chen, D. Phillips, K. Q. Schwarz, J. G. Mottley, and K. J. Parker, "The measurement of backscatter coefficient from a broadband pulseecho system: a new formulation," IEEE Transactions on Ultrasonics, Ferroelectrics, and Frequency Control, vol. 44, no. 2, pp. 515-525, March 1997.

[9] M. L. Oelze, J. F. Zachary, and W. D. O'Brien, Jr., "Characterization of tissue microstructure using ultrasonic backscatter: Theory and technique for optimization using a Gaussian form factor,' Journal of the Acoustical Society of America, vol. 112, no. 3, pp. 1202-1211, September 2002

[10] P. Chaturvedi and M. F. Insana, "Error bounds on ultrasonic scatterer size estimates," Journal of the Acoustical Society of America, vol. 100, no. 1, pp. 392-399, July 1996. 